\title{
The biodiversity of the marine Heterobranchia fauna along the central-eastern coast of Sicily, Ionian Sea
}

\author{
Andrea Lombardo* \& Giuliana Marletta \\ Department of Biological, Geological and Environmental Sciences - Section of Animal Biology, University of \\ Catania, via Androne 81, 95124 Catania, Italy \\ ${ }^{*}$ Corresponding author: andylombardo94@gmail.com
}

\begin{abstract}
The first updated list of the marine Heterobranchia for the central-eastern coast of Sicily (Italy) is here reported. This study was carried out, through a total of 271 scuba dives, from 2017 to the beginning of 2020 in four sites located along the Ionian coasts of Sicily: Catania, Aci Trezza, Santa Maria La Scala and Santa Tecla. Through a photographic data collection, 95 taxa, representing $17.27 \%$ of all Mediterranean marine Heterobranchia, were reported. The order with the highest number of found species was that of Nudibranchia. Among the study areas, Catania, Santa Maria La Scala and Santa Tecla had not a remarkable difference in the number of species, while Aci Trezza had the lowest number of species. Moreover, among the 95 taxa, four species considered rare and six non-indigenous species have been recorded. Since the presence of a high diversity of sea slugs in a relatively small area, the central-eastern coast of Sicily could be considered a zone of high biodiversity for the marine Heterobranchia fauna.
\end{abstract}

KEY WORDS diversity; marine Heterobranchia; Mediterranean Sea; sea slugs; species list.

Received 08.07.2020; accepted 08.10.2020; published online 20.11.2020

\section{INTRODUCTION}

Malacological studies and researches on marine Heterobranchia in Sicily (Italy) have always been scarce and fragmentary. Indeed, the last study on Sicilian marine Heterobranchia dates back to 1987 (Cattaneo Vietti \& Chemello, 1987). Cattaneo and Barletta (1984) argued that taxa collected and reported in Sicily represent about $30 \%$ of all Mediterranean species. This last date may not reflect a low level of biodiversity in marine Heterobranchia, but a lack of data on this group (Cattaneo Vietti \& Chemello, 1987). Moreover, studies on sea slugs in Sicily show a difference. Indeed, the eastern sector was less explored than the western sector, where more researches were carried out (Cattaneo Vietti \& Chemello, 1987).

In this work the marine Heterobranchia fauna of the central-eastern sector of Sicily (Ionian Sea) has been studied. This area, represented by Etna's eastern slopes, is geologically constituted by a complex of subvolcanic rocks, mainly consisting of columnar basalts deposited within the "pre-Etnean Gulf", and effusive marine products forming fields of pillow lavas (Cristofolini, 1975; Corsaro \& Cristofolini, 1997; Sciuto et al., 2017). Accumulated along the coast and at the base of shallow cliffs, there is a belt of blocks up to a few meters in size, caused by rocky outcrops dissected by fault systems and partially dismantled (Sciuto et al., 
2017). From the coastline to down to $10-15 \mathrm{~m}$ depth, the bottom has a sloping topography and consists of the basaltic bedrock covered with large volcanic blocks (Sciuto et al., 2017). Offshore, the bottom of sediments becomes progressively muddier at major depths with rocky outcrops locally exposed (Rosso, 2001). The study area hosts several benthic habitats, some of which are protected by the EU Habitat Directive (92/43/EEC): precoralligenous and coralligenous assemblages, softbottom substrates, Posidonia oceanica (Linnaeus) Delile, 1813 meadows, and algal biocoenosis on rocky substrates.

To carry out this study six stations along the central-eastern coast of Sicily were selected, two of which are in Protected Areas. Indeed, in the site of Aci Trezza (37.561389 N; 15.157492 E) there are a Marine Protected Area (MPA) named "Ciclopi Islands", an Integral Nature Reserve called "Lachea Island and Cyclops stacks" and two Sites of Community Importance (SCIs) ("Bottoms of Acicastello" and "Cyclops Islands"), according to the Natura 2000 network. Furthermore, in the site of Santa Maria La Scala (37.617222 N; 15.172222 E) there are an Oriented Natural Reserve called "La Timpa" and a Site of Community Importance named "Timpa of Acireale" (Catra et al., 2006). Therefore, especially in these areas, knowing and preserving the biodiversity of sea slugs is extremely important and necessary. Indeed, the changes in marine Heterobranchia fauna composition may reveal environmental modifications (Furfaro et al., 2020), caused by natural or anthropogenic factors. Moreover, a knowledge on the diversity of the marine Heterobranchia fauna can disclose the presence of any non-indigenous species. Consequently, studies focused on the production of species lists allow the hidden diversity to be revealed (Furfaro et al., 2020) and allow comparisons with different geographical areas to be made.

To date, there is not an updated list on the sea slugs of the central-eastern sector of Sicily. Indeed, data on the marine Heterobranchia fauna of the central-eastern sector of Sicily, referred to as Gulf of Catania and provinces, are ancient and reported only 5 species (Philippi, 1836-1844; Aradas, 1847; Cattaneo Vietti \& Chemello, 1987). For this reason, the aim of this work was to contribute to the knowledge of the marine Heterobranchia present in this area, which has always been poorly explored, and to document the presence of rare and non-indigenous species.

\section{MATERIAL AND METHODS}

This study was carried out from January 2017 to March 2020, in 6 stations located along the central-eastern sector of Sicily (Figs. 1, 2). These sites were selected based on different environmental conditions. Two stations, "Ognina" (37.530528 N; 15.120028 E) and "Bellatrix" (37.533917 N; $15.126778 \mathrm{E})$, are situated close to each other in the municipality of Catania. These sites are sub-

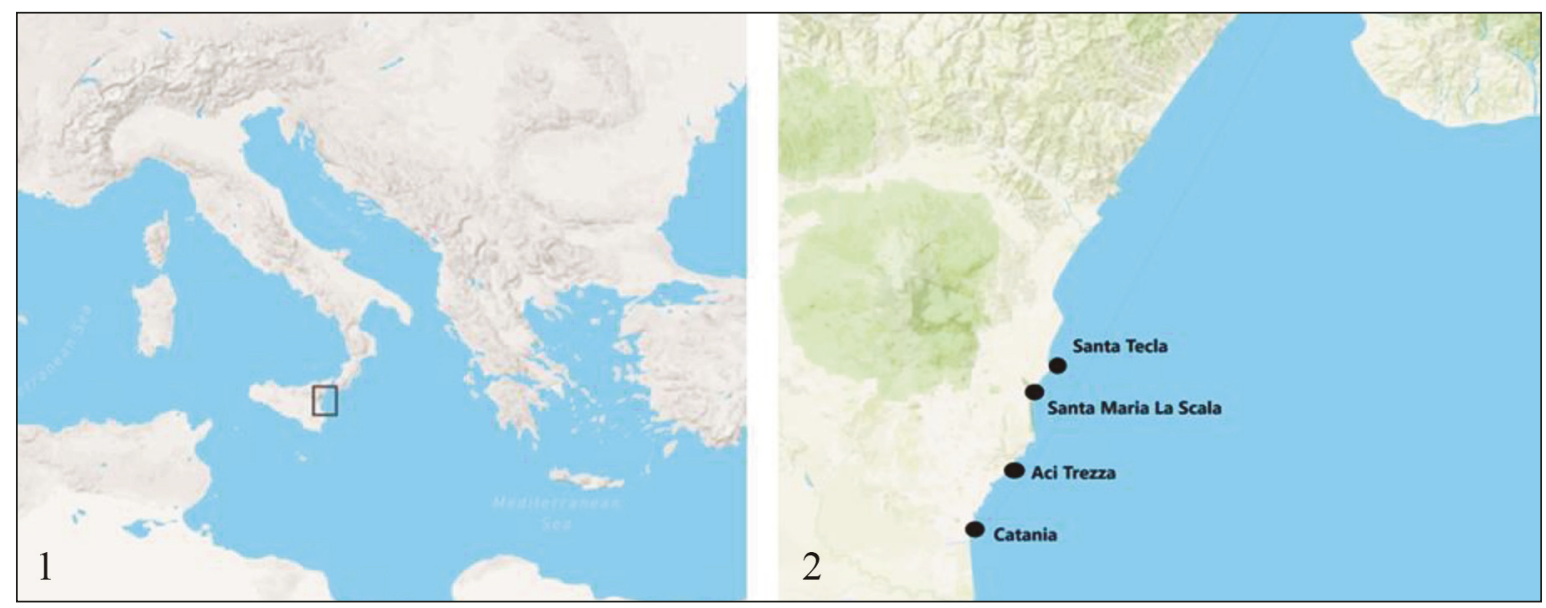

Figures 1, 2. Geographical area in the Ionian Sea (Mediterranean Sea) where this study was carried out: central-eastern sector of Sicily, where are located the study areas (Fig. 1); detail of the study areas (Fig. 2). 
jected to a strong anthropization, due to the proximity of a harbour and the exploitation of the coast. Since these sites have similar ecological conditions, they were considered as a single site, listed from now on as "Catania". Another station called "Padre Pio" (37.566083 N; 15.166361 E) is located in the zone "B", the general reserve zone, of the MPA of "Ciclopi Islands", in the hamlet of Aci Trezza, within the municipality of Acicastello. This site, especially during summer, has a strong anthropic pressure. From now on, for simplification this area will be referred as "Aci Trezza". A station named "Testa dell'acqua" (37.6125 N; 15.175389 E) is located in the hamlet of Santa Maria La Scala and, thus, it will be simply listed as "Santa Maria La Scala". Finally, two sites, "Scalo Pennisi" (37.639778 N; 15.184722 E) and "Acque Fredde" $(37.637583 \mathrm{~N} ; 15.181167 \mathrm{E})$, are situated in the hamlet of Santa Tecla. These three stations are all located in the municipality of Acireale. Moreover, in these three sites there are several springs due to the flow of freshwater from the Etna to the sea (Ferrara, 1977; Catra et al., 2006). Since the stations of "Scalo Pennisi" and "Acque Fredde" are situated close to each other and are the least affected by anthropogenic factors, they considered as a single site, listed from now on as "Santa Tecla". Instead, the site of "Santa Maria La Scala" was considered individually because it has intermediate conditions between those of Catania and Santa Tecla.

Throughout the years of study, a total of 271 scuba dives was carried out: 92 in "Catania", 81 in "Santa Maria La Scala", 74 in "Santa Tecla", 24 in "Aci Trezza". The scuba dives $(0-45 \mathrm{~m})$ were conducted all year round (marine-weather conditions permitting), twice a week, during daylight, between 9-11:30 am. Occasionally, some dives were carried out at night in the site of Aci Trezza. In each site, the same pathway has always been followed. Data on marine Heterobranchia fauna were collected with the "photographic capture" technique: during each scuba dive all sea slugs were photographed with an Olympus TG-4 Underwater Camera, were counted and the depth, where they were encountered, was annotated. Subsequently, the photographs with the specimens were used for the identification of the species, by consulting Schmekel \& Portmann (1982), Thomson \& Brown (1984), Betti (2011), Trainito \& Doneddu
(2014) and Sea Slug Forum (Rudman, 1999). The validity of the species names has been checked by consulting the World Register of Marine Species (Horton et al., 2020). Finally, the collected data were processed in Excel to create a table and graphs.

\section{RESULTS}

Through data collection, a total of 95 taxa of marine Heterobranchia (Table 1) were found, consisting of: 1 Rhodopoidea, 4 Pleurobranchida, 61 Nudibranchia, 2 Umbraculida, 7 Aplysiida, 5 Cephalaspidaea, 1 Runcinida, 14 Sacoglossa. Their percentages are shown in figure 3. Among these 95 taxa, 75 were found in Catania, 43 in Aci Trezza, 74 in Santa Maria La Scala, 71 in Santa Tecla (Fig. 4).

Since the highest percentage of species belongs to the order Nudibranchia (64\%), the differences within this order have been highlighted and it has been seen that $56 \%$ of them are represented by the suborder Cladobranchia and $44 \%$ by the suborder Doridina. Within the suborder Cladobranchia (Fig. 5 ), a total of 34 species was found, consisting of 12 families. Instead, within the suborder Doridina (Fig. 6) a total of 27 species was found, consisting of 9 families.

In Table 1 all sea slug species, which were found throughout this study, are reported. The asterisks present in the table represent the finding of only the eggs of a species in an area.

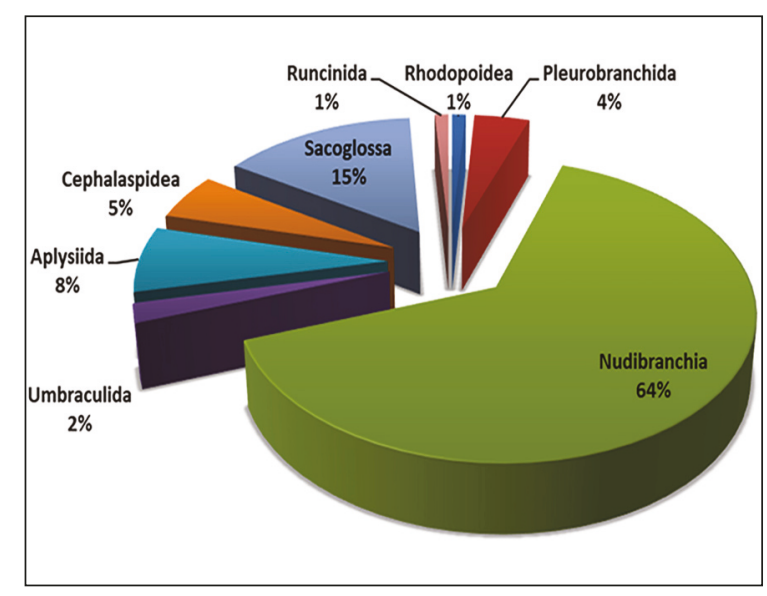

Figure 3. Percentages of the marine Heterobranchia groups found throughout the study. 


\begin{tabular}{|c|c|c|c|c|c|}
\hline Taxa & Catania & Acitrezza & $\begin{array}{c}\text { Santa } \\
\text { Maria La } \\
\text { Scala }\end{array}$ & Santa Tecla & Depth \\
\hline \multicolumn{6}{|l|}{ "Lower Heterobranchia" } \\
\hline \multicolumn{6}{|l|}{ Superfamily Rhodopoidea Ihering, 1876} \\
\hline \multicolumn{6}{|l|}{ Family Rhodopidae Ihering, 1876} \\
\hline Rhodope sp. & $\mathbf{X}$ & & $\mathbf{X}$ & $\mathbf{X}$ & $6.8-28 \mathrm{~m}$ \\
\hline \multicolumn{6}{|l|}{ Euthyneura } \\
\hline \multicolumn{6}{|l|}{ Order Pleurobranchida } \\
\hline \multicolumn{6}{|l|}{ Family Pleurobranchidae Gray, 1827} \\
\hline Berthella $\mathrm{cf}$. aurantiaca (Risso, 1818) & $\mathbf{X}$ & & & & $6 \mathrm{~m}$ \\
\hline Berthella ocellata (Delle Chiaje, 1830) & $\mathbf{X}$ & $\mathbf{X}$ & & $\mathbf{X}$ & $15-34.2 \mathrm{~m}$ \\
\hline Pleurobranchus testudinarius Cantraine, 1835 & $\mathbf{X}$ & $\mathbf{X}$ & * & * & $5-45 \mathrm{~m}$ \\
\hline Pleurobranchus wirtzi Ortea, Moro \& Caballer, 2014 & $\mathbf{X}$ & & & & $6.6 \mathrm{~m}$ \\
\hline \multicolumn{6}{|l|}{ Order Nudibranchia } \\
\hline \multicolumn{6}{|l|}{ Suborder Doridina } \\
\hline \multicolumn{6}{|l|}{ Family Calycidorididae Roginskaya, 1972} \\
\hline Diaphorodoris alba Portmann \& Sandmeier, 1960 & $\mathbf{X}$ & $\mathbf{X}$ & $\mathbf{X}$ & $\mathbf{X}$ & $12-37 \mathrm{~m}$ \\
\hline Diaphorodoris papillata Portmann \& Sandmeier, 1960 & $\mathbf{X}$ & $\mathbf{X}$ & $\mathbf{X}$ & $\mathbf{X}$ & $7-37 \mathrm{~m}$ \\
\hline \multicolumn{6}{|l|}{ Family Goniodorididae H. Adams \& A. Adams, 1854} \\
\hline Okenia problematica Pola, Paz-Sedano, Macali et al., 2019 & $\mathbf{X}$ & & & & 14.5-17.4 m \\
\hline Trapania lineata Haefelfinger, 1960 & $\mathbf{X}$ & & $\mathbf{X}$ & $\mathbf{X}$ & $5.3-35 \mathrm{~m}$ \\
\hline \multicolumn{6}{|l|}{ Family Polyceridae Alder \& Hancock, 1845} \\
\hline Kaloplocamus ramosus (Cantraine, 1835) & & $\mathbf{X}$ & & & $15 \mathrm{~m}$ \\
\hline Polycera quadrilineata (O.F. Müller, 1776) & $\mathbf{X}$ & $\mathbf{X}$ & $\mathbf{X}$ & $\mathbf{X}$ & 6-37 m \\
\hline \multicolumn{6}{|l|}{ Family Aegiridae P. Fischer, 1883} \\
\hline Aegires leuckartii Vérany, 1853 & $\mathbf{X}$ & & & $\mathbf{X}$ & 6-17.1 m \\
\hline Aegires sublaevis Odhner, 1932 & $\mathbf{X}$ & & & & $5,7 \mathrm{~m}$ \\
\hline \multicolumn{6}{|l|}{ Family Chromodorididae Bergh, 1891} \\
\hline Felimare fontandraui (Pruvot-Fol, 1951) & $\mathbf{X}$ & $\mathbf{X}$ & $\mathbf{X}$ & $\mathbf{X}$ & 9-37 m \\
\hline Felimare gasconi (Ortea, 1996) & $\mathbf{X}$ & & $\mathbf{X}$ & & 6.1-19.2 m \\
\hline Felimare orsinii (Vérany, 1846) & & & $\mathbf{X}$ & & $25,6 \mathrm{~m}$ \\
\hline Felimare picta (Philippi, 1836) & $\mathbf{X}$ & $\mathbf{X}$ & $\mathbf{X}$ & $\mathbf{X}$ & 4-36.6 m \\
\hline Felimare tricolor (Cantraine, 1835) & $\mathbf{X}$ & $\mathbf{X}$ & $\mathbf{X}$ & $\mathbf{X}$ & 7-37.2 m \\
\hline Felimare villafranca (Risso, 1818) & $\mathbf{X}$ & & $\mathbf{X}$ & $\mathbf{X}$ & 8-30 m \\
\hline Felimida binza (Ev. Marcus \& Er. Marcus, 1963) & $\mathbf{X}$ & $\mathbf{X}$ & $\mathbf{X}$ & $\mathbf{X}$ & 6-34 m \\
\hline Felimida krohni (Vérany, 1846) & $\mathbf{X}$ & $\mathbf{X}$ & $\mathbf{X}$ & $\mathbf{X}$ & 6-36 m \\
\hline Felimida luteorosea (Rapp, 1827) & $\mathbf{X}$ & & $\mathbf{X}$ & $\mathbf{X}$ & 10-35 m \\
\hline Felimida purpurea (Risso, 1831) & $\mathbf{X}$ & & $\mathbf{X}$ & $\mathbf{X}$ & $8-35.5 \mathrm{~m}$ \\
\hline
\end{tabular}




\begin{tabular}{|c|c|c|c|c|c|}
\hline Taxa & Catania & Acitrezza & $\begin{array}{c}\text { Santa } \\
\text { Maria La } \\
\text { Scala }\end{array}$ & Santa Tecla & Depth \\
\hline \multicolumn{6}{|l|}{ Family Dorididae Rafinesque, 1815} \\
\hline Doris verrucosa Linnaeus, 1758 & & $\mathbf{X}$ & & & $<1 \mathrm{~m}$ \\
\hline \multicolumn{6}{|l|}{ Family Discodorididae Bergh, 1891} \\
\hline Baptodoris cf. cinnabarina Bergh, 1884 & $\mathbf{X}$ & & & & $5,7 \mathrm{~m}$ \\
\hline Jorunna tomentosa (Cuvier, 1804) & & $\mathbf{X}$ & & & $<1 \mathrm{~m}$ \\
\hline Peltodoris atromaculata Bergh, 1880 & $\mathbf{X}$ & $\mathbf{X}$ & $\mathbf{X}$ & $\mathbf{X}$ & 10-37 m \\
\hline Platydoris argo (Linnaeus, 1767) & $\mathbf{X}$ & & $\mathbf{X}$ & $\mathbf{X}$ & $<1-34 \mathrm{~m}$ \\
\hline Taringa tritorquis Ortea, Perez \& Llera, 1982 & $\mathbf{X}$ & & $\mathbf{X}$ & & $6.9-9.5 \mathrm{~m}$ \\
\hline \multicolumn{6}{|l|}{ Family Phyllidiidae Rafinesque, 1814} \\
\hline Phyllidia flava Aradas, 1847 & $\mathbf{X}$ & $\mathbf{X}$ & $\mathbf{X}$ & & $12-37 \mathrm{~m}$ \\
\hline \multicolumn{6}{|l|}{ Family Dendrodorididae O'Donoghue, 1924 (1864) } \\
\hline Dendrodoris limbata (Cuvier, 1804) & $\mathbf{X}$ & $\mathbf{X}$ & & $\mathbf{X}$ & 3.7-28.4 m \\
\hline Doriopsilla areolata Bergh, 1880 & & & $\mathbf{X}$ & $\mathbf{X}$ & 5-8.7 m \\
\hline \multicolumn{6}{|l|}{ Suborder Cladobranchia } \\
\hline \multicolumn{6}{|l|}{ Family Tritoniidae Lamarck, 1809} \\
\hline Tritonia manicata Deshayes, 1853 & & & $\mathbf{X}$ & & $34 \mathrm{~m}$ \\
\hline Tritonia striata Haefelfinger, 1963 & & $\mathbf{X}$ & & & $17 \mathrm{~m}$ \\
\hline \multicolumn{6}{|l|}{ Family Dotidae Gray, 1853} \\
\hline Doto acuta Schmekel \& Kress, 1977 & $\mathbf{X}$ & & $\mathbf{X}$ & $\mathbf{X}$ & 6-34 m \\
\hline Doto cervicenigra Ortea \& Bouchet, 1989 & $\mathbf{X}$ & & & $\mathbf{X}$ & $15-20 \mathrm{~m}$ \\
\hline Doto floridicola Simroth, 1888 & & & $\mathbf{X}$ & $\mathbf{X}$ & $17.5-32.5 \mathrm{~m}$ \\
\hline \multicolumn{6}{|l|}{ Family Janolidae Pruvot-Fol, 1933} \\
\hline Antiopella cristata (Delle Chiaje, 1841) & $\mathbf{X}$ & $\mathbf{X}$ & $\mathbf{X}$ & $\mathbf{X}$ & $11-36 \mathrm{~m}$ \\
\hline \multicolumn{6}{|l|}{ Family Flabellinidae Bergh, 1889} \\
\hline Calmella cavolini (Vérany, 1846) & $\mathbf{X}$ & $\mathbf{X}$ & $\mathbf{X}$ & $\mathbf{X}$ & 4-37 m \\
\hline Edmundsella pedata (Montagu, 1816) & $\mathbf{X}$ & $\mathbf{X}$ & $\mathbf{X}$ & $\mathbf{X}$ & 3-37 m \\
\hline Flabellina affinis (Gmelin, 1791) & $\mathbf{X}$ & $\mathbf{X}$ & $\mathbf{X}$ & $\mathbf{X}$ & $8-38 \mathrm{~m}$ \\
\hline Paraflabellina ischitana (Hirano \& T. E. Thompson, 1990) & $\mathbf{X}$ & & $\mathbf{X}$ & $\mathbf{X}$ & 5-37 m \\
\hline \multicolumn{6}{|l|}{ Family Coryphellidae Bergh, 1889} \\
\hline Fjordia lineata (Lovén, 1846) & $\mathbf{X}$ & & $\mathbf{X}$ & $\mathbf{X}$ & $8,5-34 \mathrm{~m}$ \\
\hline \multicolumn{6}{|l|}{ Family Samlidae Korshunova, Martynov et al., 2017} \\
\hline Luisella babai (Schmekel, 1972) & $\mathbf{X}$ & $\mathbf{X}$ & $\mathbf{X}$ & $\mathbf{X}$ & 20-37 m \\
\hline \multicolumn{6}{|l|}{ Family Eubranchidae Odhner, 1934} \\
\hline Amphorina farrani (Alder \& Hancock, 1844) & $\mathbf{X}$ & & $\mathbf{X}$ & $\mathbf{X}$ & 5-16 m \\
\hline Capellinia doriae Trinchese, 1874 & & & $\mathbf{X}$ & & $9.1 \mathrm{~m}$ \\
\hline Eubranchus tricolor Forbes, 1838 & & & & $\mathbf{X}$ & $9 \mathrm{~m}$ \\
\hline \multicolumn{6}{|l|}{ Family Trinchesiidae F. Nordsieck, 1972} \\
\hline Trinchesia caerulea (Montagu, 1804) & $\mathbf{X}$ & & $\mathbf{X}$ & $\mathbf{X}$ & $6.5-37 \mathrm{~m}$ \\
\hline
\end{tabular}




\begin{tabular}{|c|c|c|c|c|c|}
\hline Taxa & Catania & Acitrezza & $\begin{array}{c}\text { Santa } \\
\text { Maria La } \\
\text { Scala }\end{array}$ & Santa Tecla & Depth \\
\hline Trinchesia foliata (Forbes \& Goodsir, 1839) & $\mathbf{X}$ & $\mathbf{X}$ & $\mathbf{X}$ & $\mathbf{X}$ & $6-27 \mathrm{~m}$ \\
\hline Trinchesia genovae (O'Donoghue, 1926) & $\mathbf{X}$ & $\mathbf{X}$ & $\mathbf{X}$ & $\mathbf{X}$ & $5-35 \mathrm{~m}$ \\
\hline Trinchesia ocellata Schmekel, 1966 & $\mathbf{X}$ & & $\mathbf{X}$ & $\mathbf{X}$ & 17-36 m \\
\hline \multicolumn{6}{|l|}{ Family Embletoniidae Pruvot-Fol, 1954} \\
\hline Embletonia pulchra (Alder \& Hancock, 1844) & $\mathbf{X}$ & & $\mathbf{X}$ & $\mathbf{X}$ & $10-6 \mathrm{~m}$ \\
\hline \multicolumn{6}{|l|}{ Family Facelinidae Bergh, 1889} \\
\hline Caloria elegans (Alder \& Hancock, 1845) & $\mathbf{X}$ & & $\mathbf{X}$ & & 21.3-27.8 m \\
\hline Cratena peregrina (Gmelin, 1791) & $\mathbf{X}$ & $\mathbf{X}$ & $\mathbf{X}$ & $\mathbf{X}$ & 4-37 m \\
\hline Dicata odhneri Schmekel, 1967 & $\mathbf{X}$ & $\mathbf{X}$ & $\mathbf{X}$ & $\mathbf{X}$ & 4.5-32 m \\
\hline Facelina annulicornis (Chamisso \& Eysenhardt, 1821) & $\mathbf{X}$ & & $\mathbf{X}$ & $\mathbf{X}$ & $7-10 \mathrm{~m}$ \\
\hline Facelina rubrovittata (Costa A., 1866) & $\mathbf{X}$ & $\mathbf{X}$ & $\mathbf{X}$ & $\mathbf{X}$ & 5-32 m \\
\hline Facelinopsis marioni (Vayssière, 1888) & $\mathbf{X}$ & $\mathbf{X}$ & $\mathbf{X}$ & $\mathbf{X}$ & 3-22 m \\
\hline Favorinus branchialis (Rathke, 1806) & $\mathbf{X}$ & $\mathbf{X}$ & $\mathbf{X}$ & $\mathbf{X}$ & 5-37 m \\
\hline \multicolumn{6}{|l|}{ Family Myrrhinidae Bergh, 1905} \\
\hline Dondice banyulensis Portmann \&Sandmeier, 1960 & $\mathbf{X}$ & & $\mathbf{X}$ & $\mathbf{X}$ & $8-38 \mathrm{~m}$ \\
\hline Dondice trainitoi Furfaro \& Mariottini, 2020 & & & $\mathbf{X}$ & & $20 \mathrm{~m}$ \\
\hline Godiva quadricolor (Barnard, 1927) & & & $\mathbf{X}$ & & $7 \mathrm{~m}$ \\
\hline \multicolumn{6}{|l|}{ Family Aeolidiidae Gray, 1827} \\
\hline Berghia coerulescens (Laurillard, 1832) & $\mathbf{X}$ & & $\mathbf{X}$ & $\mathbf{X}$ & $8-36 \mathrm{~m}$ \\
\hline Berghia verrucicornis (A. Costa, 1867) & $\mathbf{X}$ & & & & $4,8 \mathrm{~m}$ \\
\hline Limenandra nodosa Haefelfinger \& Stamm, 1958 & & & $\mathbf{X}$ & $\mathbf{X}$ & $5-17 \mathrm{~m}$ \\
\hline Spurilla neapolitana (Delle Chiaje, 1841 ) & $\mathbf{X}$ & $\mathbf{X}$ & $\mathbf{X}$ & $\mathbf{X}$ & $5-20 \mathrm{~m}$ \\
\hline \multicolumn{6}{|l|}{ Order Umbraculida } \\
\hline \multicolumn{6}{|l|}{ Family Umbraculidae Dall, 1889 (1827) } \\
\hline Umbraculum umbraculum (Lightfoot, 1786) & & $\mathbf{X}$ & $\mathbf{X}$ & $\mathbf{X}$ & $5.1-36 \mathrm{~m}$ \\
\hline \multicolumn{6}{|l|}{ Family Tylodinidae Gray, 1847} \\
\hline Tylodina perversa (Gmelin, 1791) & $\mathbf{X}$ & & $\mathbf{X}$ & $\mathbf{X}$ & $12-31 \mathrm{~m}$ \\
\hline \multicolumn{6}{|l|}{ Order Aplysiida } \\
\hline \multicolumn{6}{|l|}{ Family Aplysiidae Lamarck, 1809} \\
\hline Aplysia dactylomela Rang, 1828 & $\mathbf{X}$ & $\mathbf{X}$ & $\mathbf{X}$ & $\mathbf{X}$ & $<1-19 \mathrm{~m}$ \\
\hline Aplysia depilans Gmelin, 1791 & $\mathbf{X}$ & & $\mathbf{X}$ & $\mathbf{X}$ & $2.9-23 \mathrm{~m}$ \\
\hline Aplysia fasciata Poiret, 1789 & $\mathbf{X}$ & & $\mathbf{X}$ & $\mathbf{X}$ & $2-25.3 \mathrm{~m}$ \\
\hline Aplysia parvula Mörch, 1863 & $\mathbf{X}$ & $\mathbf{X}$ & $\mathbf{X}$ & $\mathbf{X}$ & $2.9-17 \mathrm{~m}$ \\
\hline Aplysia punctata (Cuvier, 1803) & $\mathbf{X}$ & $\mathbf{X}$ & $\mathbf{X}$ & $\mathbf{X}$ & $1.4-21.7 \mathrm{~m}$ \\
\hline Notarchus punctatus Philippi, 1836 & $\mathbf{X}$ & $\mathbf{X}$ & $\mathbf{X}$ & $\mathbf{X}$ & 6-21,5 m \\
\hline Phyllaplysia cf. lafonti (P. Fischer, 1870) & $\mathbf{X}$ & & $\mathbf{X}$ & $\mathbf{X}$ & $5-18 \mathrm{~m}$ \\
\hline \multicolumn{6}{|l|}{ Order Cephalaspidea } \\
\hline Family Aglajidae Pilsbry, 1895 (1847) & & & & & \\
\hline
\end{tabular}




\begin{tabular}{|c|c|c|c|c|c|}
\hline Taxa & Catania & Acitrezza & $\begin{array}{c}\text { Santa } \\
\text { Maria La } \\
\text { Scala }\end{array}$ & Santa Tecla & Depth \\
\hline Aglaja tricolorata Renier, 1807 & & & $\mathbf{X}$ & $\mathbf{X}$ & $11.2-15 \mathrm{~m}$ \\
\hline Biuve fulvipunctata (Baba, 1938) & & & & $\mathbf{X}$ & $4.4 \mathrm{~m}$ \\
\hline Camachoaglaja africana (Pruvot-Fol, 1953) & $\mathbf{X}$ & $\mathbf{X}$ & & $\mathbf{X}$ & $<1-7 \mathrm{~m}$ \\
\hline \multicolumn{6}{|l|}{ Family Haminoeidae Pilsbry, 1895} \\
\hline Haloa sp. & $*$ & $\mathbf{X}$ & * & $\mathbf{X}$ & 3-33 m \\
\hline $\begin{array}{l}\text { Lamprohaminoea cyanomarginata } \\
\text { (Heller \& T.E. Thompson, 1983) }\end{array}$ & $\mathbf{X}$ & $\mathbf{X}$ & $\mathbf{X}$ & $\mathbf{X}$ & $5-25 \mathrm{~m}$ \\
\hline \multicolumn{6}{|l|}{ Order Runcinida } \\
\hline \multicolumn{6}{|l|}{ Family Runcinidae H. Adams \& A. Adams, 1854} \\
\hline Runcina sp. & $\mathbf{X}$ & & $\mathbf{X}$ & & $6-7.2 \mathrm{~m}$ \\
\hline \multicolumn{6}{|l|}{ Superorder Sacoglossa } \\
\hline \multicolumn{6}{|l|}{ Family Oxynoidae Stoliczka, 1868 (1847) } \\
\hline Lobiger serradifalci (Calcara, 1840) & & & $\mathbf{X}$ & $\mathbf{X}$ & 5-10 m \\
\hline Oxynoe olivacea Rafinesque, 1814 & & & & $\mathbf{X}$ & 6-7.6 m \\
\hline \multicolumn{6}{|l|}{ Family Plakobranchidae Gray, 1840} \\
\hline Bosellia mimetica Trinchese, 1891 & $\mathbf{X}$ & $\mathbf{X}$ & $\mathbf{X}$ & $\mathbf{X}$ & $1-38 \mathrm{~m}$ \\
\hline Elysia hetta Perrone, 1990 & $\mathbf{X}$ & & $\mathbf{X}$ & & $21-30 \mathrm{~m}$ \\
\hline Elysia margaritae Fez, 1962 & $\mathbf{X}$ & $\mathbf{X}$ & $\mathbf{X}$ & & 5-30 m \\
\hline Elysia rubeni Martín-Hervás, Carmona et al., 2019 & $\mathbf{X}$ & $\mathbf{X}$ & $\mathbf{X}$ & $\mathbf{X}$ & $7-21 \mathrm{~m}$ \\
\hline Elysia timida (Risso, 1818) & $\mathbf{X}$ & $\mathbf{X}$ & $\mathbf{X}$ & $\mathbf{X}$ & 1-18 m \\
\hline Elysia translucens Pruvot-Fol, 1957 & & & $\mathbf{X}$ & $\mathbf{X}$ & $6.8-7.5 \mathrm{~m}$ \\
\hline Elysia viridis (Montagu, 1804) & $\mathbf{X}$ & & $\mathbf{X}$ & $\mathbf{X}$ & $7-22 \mathrm{~m}$ \\
\hline Thuridilla hopei (Vérany, 1853) & $\mathbf{X}$ & $\mathbf{X}$ & $\mathbf{X}$ & $\mathbf{X}$ & $1-36 \mathrm{~m}$ \\
\hline \multicolumn{6}{|l|}{ Family Limapontiidae Gray, 1847} \\
\hline Placida cremoniana (Trinchese, 1892) & $\mathbf{X}$ & & & & $1.4 \mathrm{~m}$ \\
\hline Placida viridis (Trinchese, 1874) & & & & $\mathbf{X}$ & $7.2 \mathrm{~m}$ \\
\hline \multicolumn{6}{|l|}{ Family Hermaeidae H. Adams \& A. Adams, 1854} \\
\hline Hermaea bifida (Montagu, 1816) & $\mathbf{X}$ & & & $\mathbf{X}$ & 6-19 m \\
\hline Hermaea variopicta (A. Costa, 1869) & $\mathbf{X}$ & & $\mathbf{X}$ & & $7 \mathrm{~m}$ \\
\hline
\end{tabular}

Table 1. List of marine Heterobranchia found in four areas along the central-eastern sector of Sicily (Ionian Sea). Abbreviation cf. is from the Latin confer/conferatur, both meaning compare.

\section{DISCUSSION}

The present study, which was carried out from 2017 to the beginning of 2020 in four areas located along the central-eastern sector of Sicily, reports the presence of 95 taxa of marine Heterobranchia (Table 1). Through data collection, it was observed that the highest number of the found species belongs to the order Nudibranchia, with a percentage of $64 \%$ (Fig. 3). Moreover, within this order the majority of the species belongs to the suborder Cladobranchia $(56 \%)$, in which the family with the highest number of species was that of Facelinidae Bergh, 1889 (Fig. 5). Instead, the suborder Doridina represented $44 \%$ of the Nudibranchia. In this suborder, 


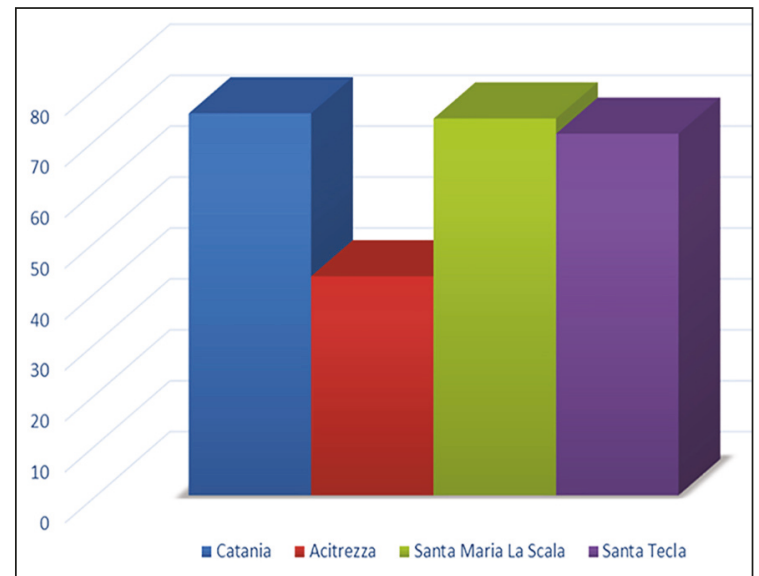

Figure 4. Number of taxa found in the different study areas.

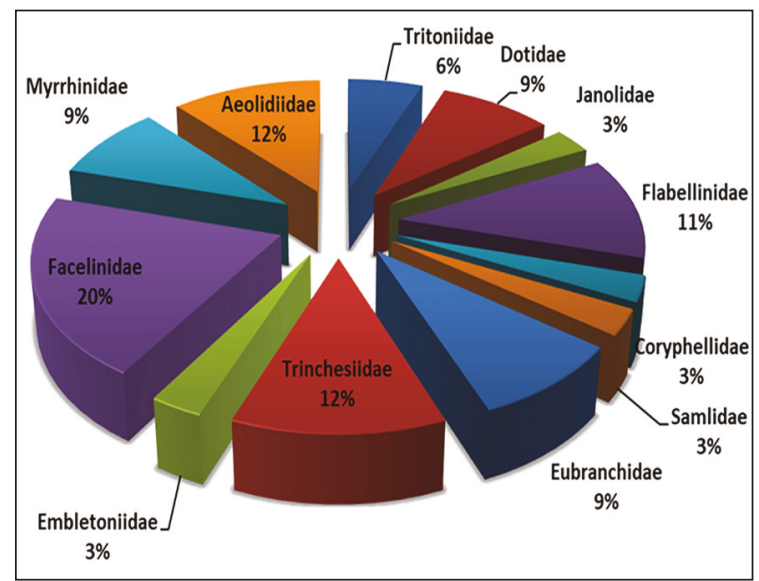

Figure 5. Percentages of the families of the suborder Cladobranchia (Nudibranchia) seen during this study.

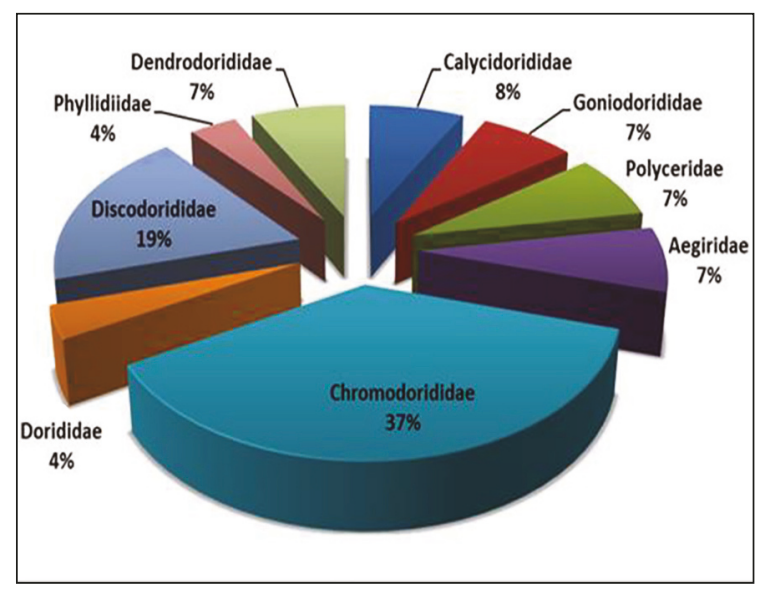

Figure 6. Percentages of the families of the suborder Doridina (Nudibranchia) seen during this study. the family with most species was that of Chromodorididae Bergh, 1891 (Fig. 6).

Regarding the distribution of the sea slug taxa in the different study areas (Fig. 4), it was not observed a remarkable difference in the number of the found species in the sites of Catania, Santa Maria La Scala and Santa Tecla. Instead, in the site of Aci Trezza the lowest number of species has been found. Although in this area fewer scuba dives were conducted than the other sites, the authors have extended knowledge of this area before the present study and believe that the lower number of species is related to other factors. Indeed, the site of Aci Trezza presents a homogeneous topography and is characterized by shallower sea bottoms, while the remaining study areas have a sloping topography and deeper sea bottoms. Therefore, this last type of topography allows the presence of a higher number of microhabitats and more variable environmental conditions.

Through data collection, for the first time it has been realized a list of the marine Heterobranchia present in the central-eastern coast of Sicily. Among the 95 taxa here reported, four species considered rare and five non-indigenous species have been found. Among the rare species, the following are reported: Elysia rubeni Martín-Hervás, Carmona, K. R. Jensen, Licchelli, Vitale \& Cervera, 2019 (Gerovasileiou et al., 2020), Okenia problematica Pola, Paz-Sedano, Macali, Minchin, Marchini,Vitale, Licchelli et Crocetta, 2019 (Lombardo \& Marletta, 2020a), Taringa tritorquis Ortea, Perez \& Llera, 1982 (Gerovasileiou et al., 2020; Lombardo \& Marletta, 2020b) and Dondice trainitoi Furfaro \& Mariottini, 2020 (Gerovasileiou et al., 2020). Instead, regarding non-indigenous species, the following taxa are here reported: Aplysia dactylomela Rang, 1828 (Valdés et al., 2013), Lamprohaminoea cyanomarginata (Heller \& T. E. Thompson, 1983) (Crocetta \& Vazzana, 2009), Godiva quadricolor (Barnard, 1927) (Lombardo \& Marletta, 2019a), Biuve fulvipunctata (Baba, 1938) (Lombardo \& Marletta, 2020b), and Pleurobranchus wirtzi Ortea, Moro \& Caballer, 2014 (Lombardo \& Marletta, 2019b). Moreover, six species, whose identification is doubtful, are reported in Table 1 and in figures 7-12: Rhodope sp., Berthella $\mathrm{cf}$. aurantiaca (Risso, 1818), Baptodoris cf. cinnabarina Bergh, 1884, Phyllaplysia cf. lafonti (P. Fischer, 1870), Runcina sp., Haloa sp.

In conclusion, the present study on the marine 

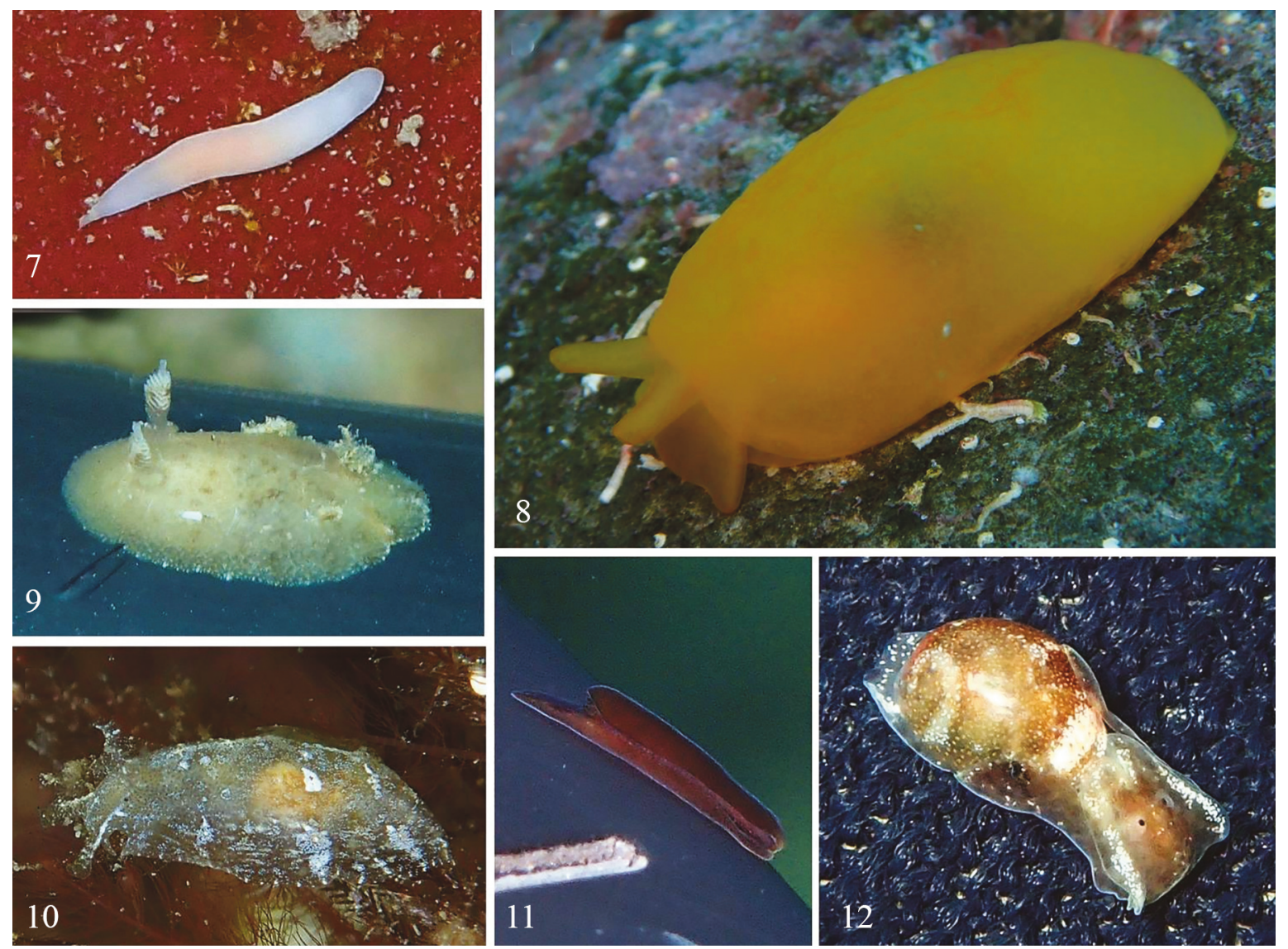

Figures 7-12. Species whose identification is doubtful: Fig. 7) Rhodope sp.; Fig. 8) Berthella cf. aurantiaca; Fig. 9) Baptodoris cf. cinnabarina; Fig. 10) Phyllaplysia cf. lafonti; Fig. 11) Runcina sp.; Fig. 12) Haloa sp. (photos A. Lombardo).

Heterobranchia of the central-eastern coast of Sicily, represents the first contribution to the knowledge of this group in this area, that has always been poorly studied. Moreover, this study revealed high biodiversity in the marine Heterobranchia present in this area. Indeed, the found taxa represent $17.27 \%$ of all Mediterranean sea slugs, which in total are about 550 (Trainito \& Doneddu, 2014; Furfaro et al., 2020). Therefore, although the small examined area, the central-eastern coast of Sicily could be considered a zone of high biodiversity for the marine Heterobranchia fauna. Future studies will be focused on the frequency, abundance and seasonality of the species distributed in the examined areas.

\section{REFERENCES}

Aradas A., 1847. Memoria $3^{\text {a }}$ cMhe contiene la de- scrizione di alcuni molluschi nudi della Sicilia. Atti Accademia Gioenia, 4: 107-122.

Betti F., 2011. Il Regno Dei Nudibranchi, guida ai molluschi opistobranchi della Riviera del Conero. Editrice La Mandragora., Imola, Italy, 198 pp.

Catra M., Giaccone T., Giardina S. \& Nicastro A., 2006. Il patrimonio naturale marino bentonico della Timpa di Acireale (Catania). Bollettino dell'Accademia Gioenia di Scienze Naturali, 39 (366): 129-158.

Cattaneo Vietti R. \& Chemello R., 1987. Alcune considerazioni sui molluschi opistobranchi della Sicilia. Bollettino Malacologico, 23: 207-222.

Cattaneo R. \& Barletta G., 1984. Elenco preliminare dei Molluschi Opistobranchi viventi nel Mediterraneo (Sacoglossa, Pleurobranchomorpha, Acochlidiacea, Aplysiomorpha, Nudibranchia). Bollettino Malacologico, 20: 195-218.

Corsaro R.A. \& Cristofolini R., 1997. Geology, geochemistry and mineral chemistry of tholeiitic to transitional etnean magmas. Acta Vulcanologica, 9: 55-66.

Cristofolini R., 1975. La massa subvulcanica di Aci 
Trezza (Etna). Rendiconti della Società Italiana di Mineralogia e Petrografa, 30: 741-770.

Crocetta F. \& Vazzana A., 2009. First record of Haminoea cyanomarginata (Gastropoda: Haminoeidae) in the Italian seas. Marine Biodiversity Records, 2: E11. https://doi.org/10.1017/S1755267208000110

Ferrara V., 1977. Aspetti idrogeologici del territorio del comune di Acireale. Memorie e rendiconti dell'Accademia di Scienze, Lettere e Belle Arti degli Zelanti e dei Dafnei, Acireale, Serie II (7): 455-488.

Furfaro G., Vitale F., Licchelli C. \& Mariottini P., 2020. Two Seas for One Great Diversity: Checklist of the Marine Heterobranchia (Mollusca; Gastropoda) from the Salento Peninsula (South-East Italy). Diversity, 12: 171. https://doi.org/10.3390/d12050171

Gerovasileiou V., Akyol O., Al-Hosne Z., Alshikh Rasheed R., Ataç E., Bello G., Ćetković I., CorsiniFoka M., Crocetta F., Denitto F., Guidetti P., Gül B., Insacco G., Jimenez C., Licchelli C., Lipej L., Lombardo A., Mancini E., Marletta G., Michailidis N., Pešić A., Poursanidis D., Refes W., Sahraoui H., Thasitis I., Tiralongo F., Tosunoğlu Z., Trkov D., Vazzana A. \& Zava B., 2020. New records of rare species in the Mediterranean Sea (May 2020). Mediterranean Marine Science, 0: 340-359. doi:https://doi.org/ $10.12681 / \mathrm{mms} .22148$

Horton T., Kroh A., Ahyong S., Bailly N., Boyko, C.B. et al., 2020. Word Register of Marine Species. http://www.marinespecies.org/ (Accessed 2 May 2020)

Lombardo A. \& Marletta G., 2019a. Presence of the alien species Godiva quadricolor (Barnard, 1927) (Gastropoda Nudibranchia Facelinidae) along the coast of eastern Sicily. Biodiversity Journal, 10: 147-150. https://doi.org/10.31396/Biodiv.Jour.2019.10.2.147.1 50

Lombardo A. \& Marletta G., 2019b. A new Atlantic immigrant in the Mediterranean Sea: Pleurobranchus wirtzi Ortea, Moro et Caballer, 2014 (Gastropoda Pleurobranchida). Biodiversity Journal, 10: 275-278. https://doi.org/10.31396/Biodiv.Jour.2019.10.3.275.2 78

Lombardo A. \& Marletta G., 2020a. First record of Oke- nia problematica Pola, Paz-Sedano, Macali, Minchin, Marchini, Vitale, Licchelli et Crocetta, 2019 (Gastropoda Nudibranchia Goniodorididae) for Sicily (Ionian Sea, Italy). Biodiversity Journal, 11: 17-20. https://doi.org/10.31396/Biodiv.Jour.2020.11.1.17.20

Lombardo A. \& Marletta G., 2020b. New records of Biuve fulvipunctata (Baba, 1939) (Gastropoda: Cephalaspidea) and Taringa tritorquis Ortea, Perez \& Llera, 1982 (Gastropoda: Nudibranchia) in the Ionian coasts of Sicily, Mediterranean Sea. Biodiversity Journal (in press).

Philippi R.A., 1836-1844. Enumeratio Molluscorum siciliae ecc,, vol.. I and II, Berolini.

Rosso A., 2001. Cartografa topografica e biocenotica dei fondali mobili della Riserva Marina "Isole Ciclopi". Scientifc report. 119 pp. Ministero dell'Ambiente Capitaneria di Porto - Università degli Studi di Catania, Catania.

Rudman W.B., 1999. Sea Slug Forum. Australian Museum, Sydney. http://www.seaslugforum.net/ (Accessed 1 May 2020).

Schmekel L. \& Portmann A., 1982. Opisthobranchia des Mittelmeeres. Nudibranchia und Saccoglossa. Springer-Verlag., Berlin, Germany, 410 pp.

Sciuto F., Sanfilippo R., Alongi G., Catra M., Serio D., Bejaoui S., Leonardi R., Viola A. \& Rosso A., 2017. First data on ostracods and foraminifera living in Cystoseira communities in western Ionian Sea (southern Italy, Mediterranean Sea). Mediterranean Marine Science, 18: 393-405. https://doi.org/10. 12681/mms.2085

Thompson T.E. \& Brown G.H., 1984. Biology of Opisthobranchs molluscs, Volume 2. The Ray Society., London, United Kingdom, 229 pp.

Trainito E. \& Doneddu M., 2014. Nudibranchi del Mediterraneo. Il Castello., Cornaredo, Italy, 192 pp.

Valdes Á., Alexander J., Crocetta F., Yokes M.B., Giacobbe S., Poursanidis D., Zenetos A., Cervera J.L., Caballer M., Galil B.S. \& Schembri P.J., 2013. The origin and dispersal pathway of the spotted sea hare Aplysia dactylomela (Mollusca: Opisthobranchia) in the Mediterranean Sea. Aquatic Invasions, 8: 427e436. http://dx.doi.org/10.3391/ai.2013.8.4.06 